\title{
LA-UR-19-27283
}

Approved for public release; distribution is unlimited.

Title: Is There A Solar Model Solution to The Faint Young Sun Paradox?

Author(s): $\quad$ Farag, Ebraheem Khaled

Intended for: $\quad$ For General Presentation purposes at other Universities and institutions.

Issued: 
Disclaimer:

Los Alamos National Laboratory, an affirmative action/equal opportunity employer, is operated by Triad National Security, LLC for the National Nuclear Security Administration of U.S. Department of Energy under contract 89233218CNA000001. By approving this article, the publisher recognizes that the U.S. Government retains nonexclusive, royalty-free license to publish or reproduce the published form of this contribution, or to allow others to do so, for U.S. Government purposes. Los Alamos National Laboratory requests that the publisher identify this article as work performed under the auspices of the U.S. Department of Energy. Los Alamos National Laboratory strongly supports academic freedom and a researcher's right to publish; as an institution, however, the Laboratory does not endorse the viewpoint of a publication or guarantee its technical correctness. 


\section{Is There A Solar Model Solution to The Faint Young Sun Paradox?}

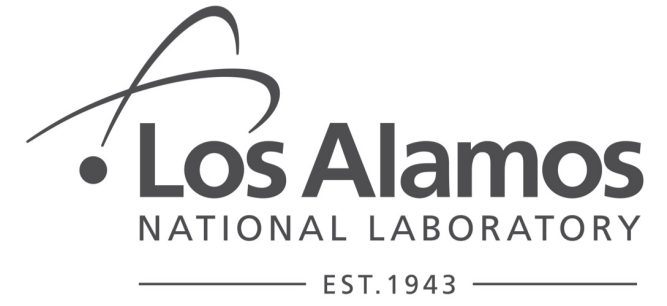

The OHIo State UnIVERSITY
Ebraheem K Farag

XTD-IDA

July $11^{\text {th }}, 2019$

Advisor: Marc Pinsonneault 


\section{Outline:}

- Faint Young Sun Paradox (FYSP)

- A higher mass Sun

- Stellar activity relations

- Angular momentum (AM) formulation

- Predicted rotation and mass loss histories of the Sun

- What our results mean for FYSP

- Questions? 


\section{The Faint Young Sun Paradox? (FYSP)}

Inability for advanced atmospheric models and solar models to generate the conditions for liquid water on the early

Earth and Mars 


\section{Liquid ocean covered Earth}

- Geologic evidence points to the Earth's oceans existing in liquid form as early as $3.8-2.5$ billion years ago

(Grotzinger, \& Kasting 1993)(Knoll et al. 2016).

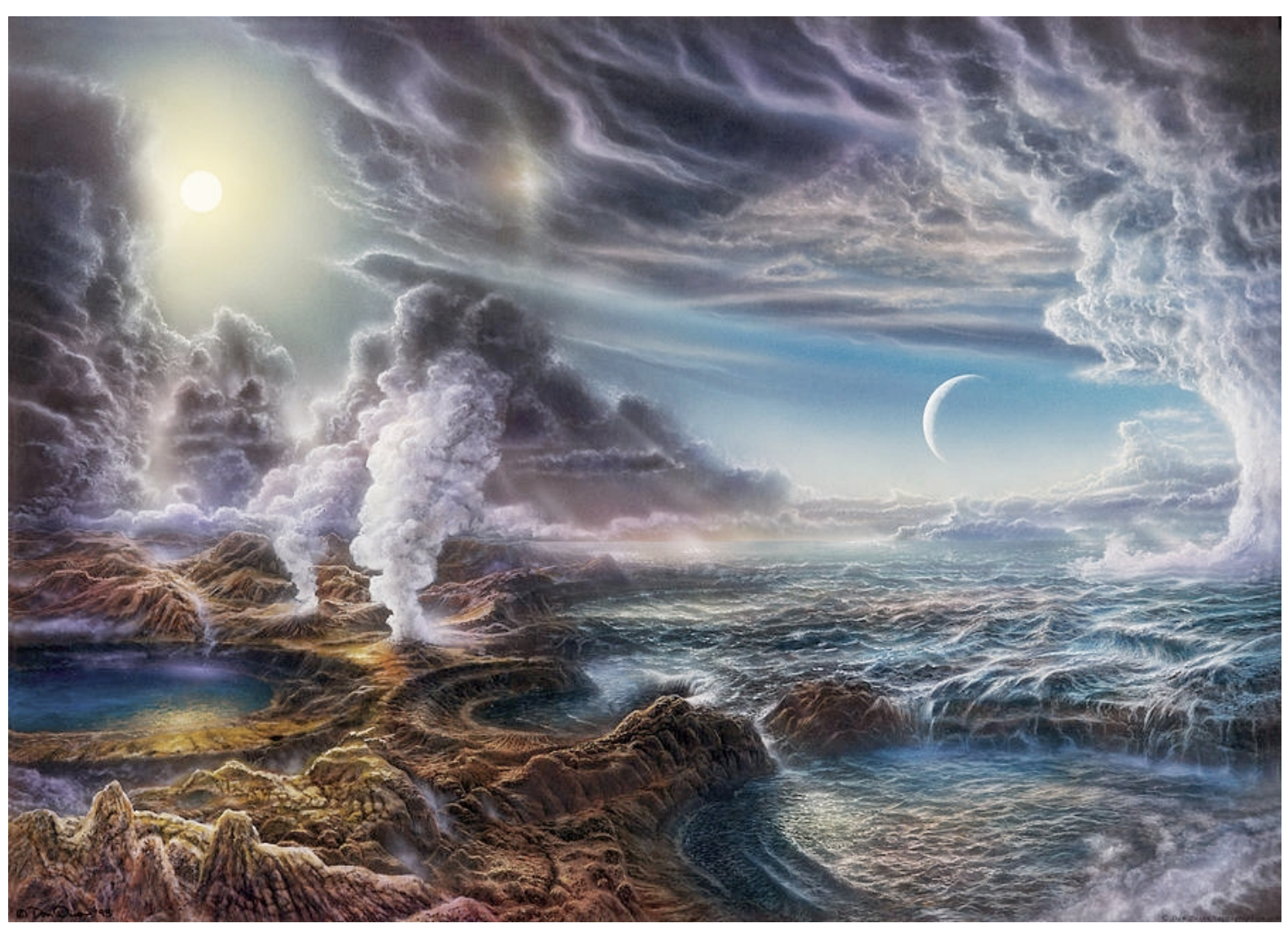

Artist's view of the young Earth as it is believed to have looked 3.5 billion years ago Image: NASA GSFC 


\section{Water carved valleys on Mars}

- Water carved valleys and sedimentary lake basins up to 4 billion years old indicate the presence of liquid water on the Martian surface

(Wordsworth 2016),

(Grotzinger et al. 2015), (Dibiase et al. 2013)
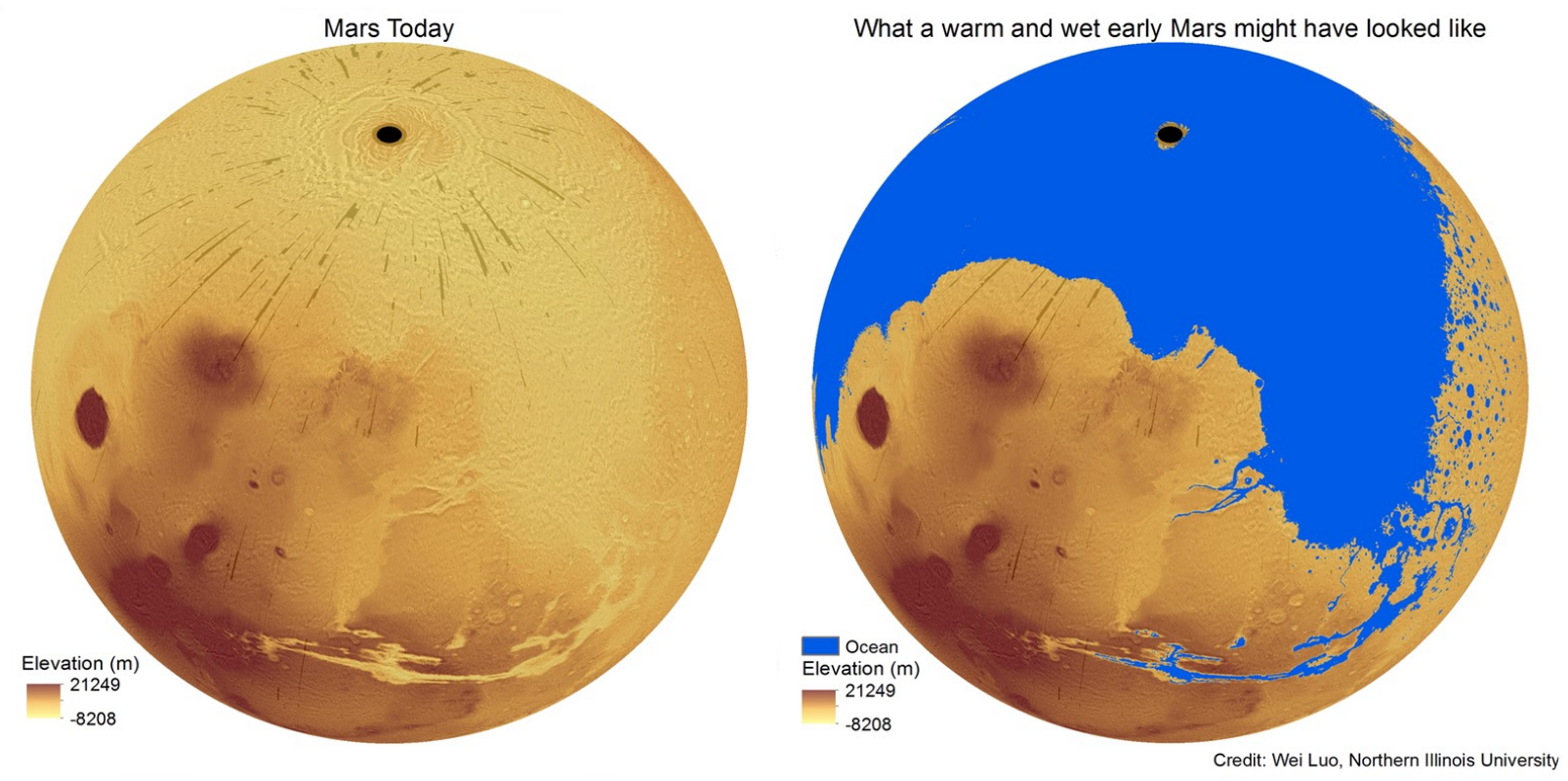


\section{The luminosity of the Sun is insensitive to changes in physics}

- Opacities

- Heavy element abundances

- Nuclear reaction rates

- Equation of state

- Diffusion

- Convective treatment

- Other microphysics etc.

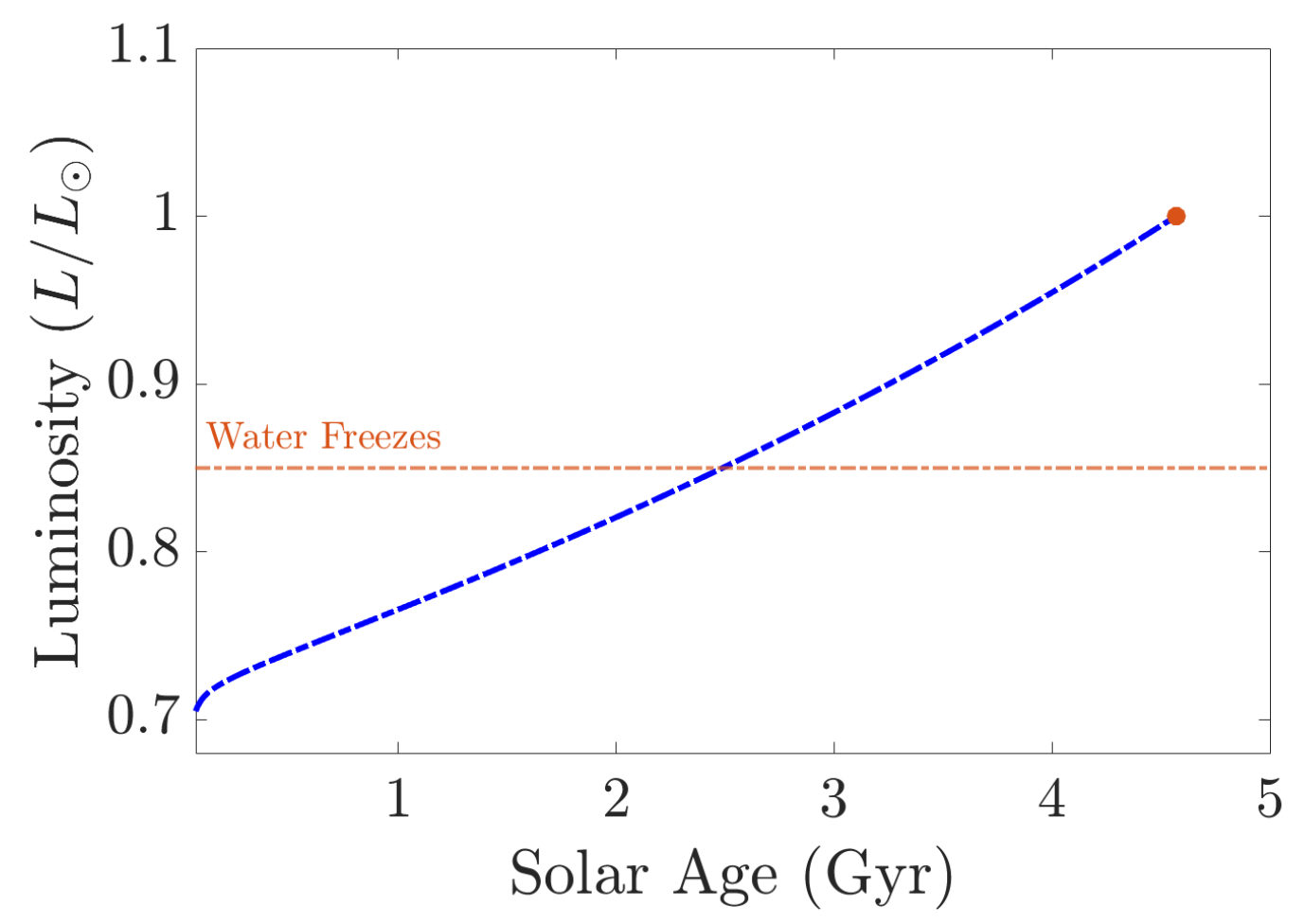

(Bahcall et al. 2001) 


\section{Two possible solutions}

- The Sun was much more massive early in its history

\section{OR}

- Better understanding of the atmosphere on early Earth and Mars 


\section{Two possible solutions}

- The Sun was much more massive early in its history

OR

- Better understanding of the atmosphere on early Earth and Mars 


\section{What if the Sun was more massive?}

Mass-Luminosity Relation

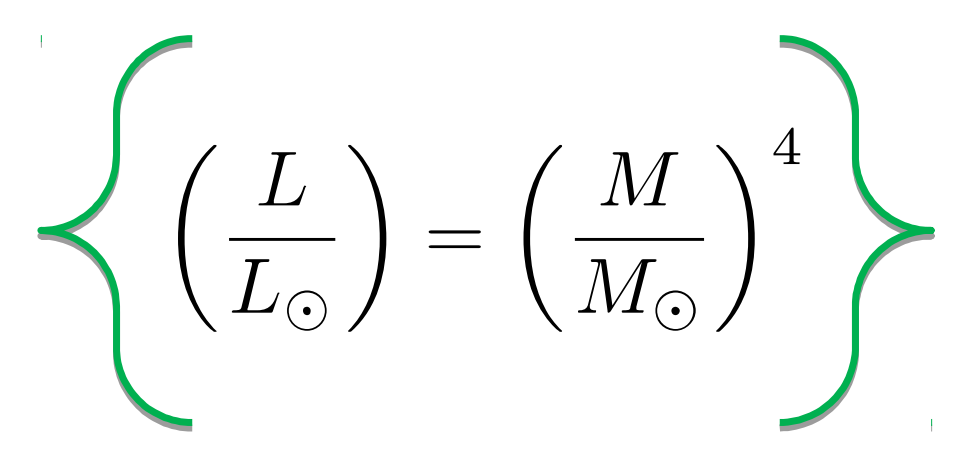

-5-6\% More Mass

20-26\% Higher Luminosity 


\section{What if the Sun was more massive?}
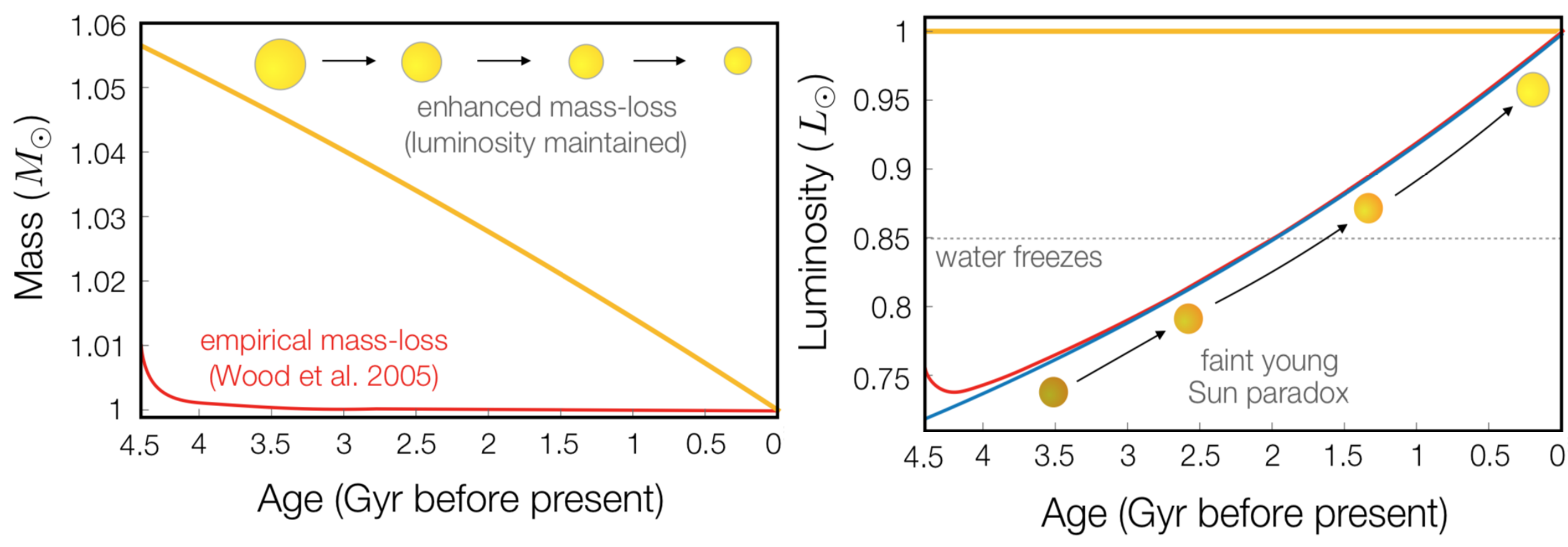

(Spalding et al. 2018),(Suzannah Wood et al. 2018) 


\section{What drives mass loss?}

Nasa.gov

Stellar Winds

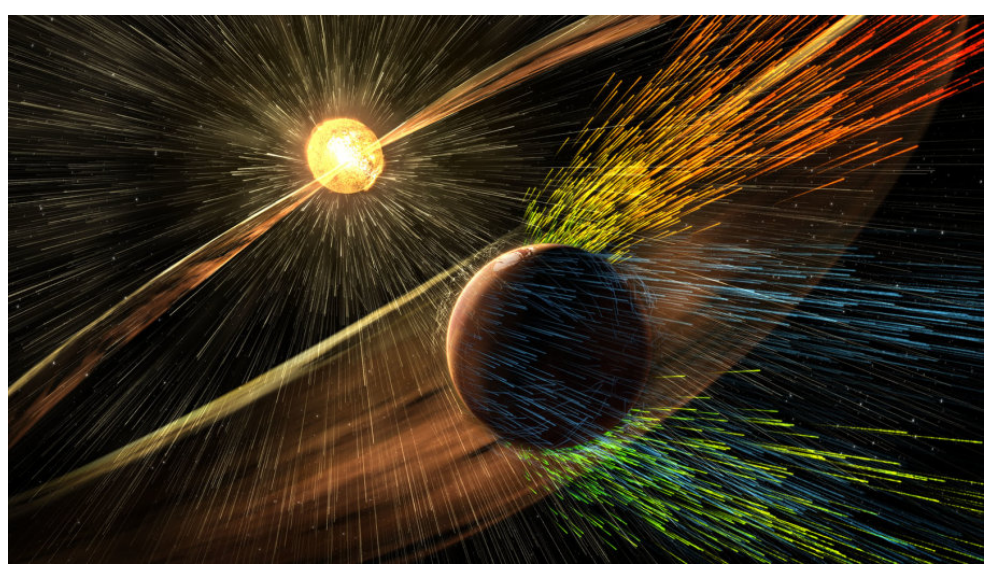

Coronal Mass Ejections (CMEs)

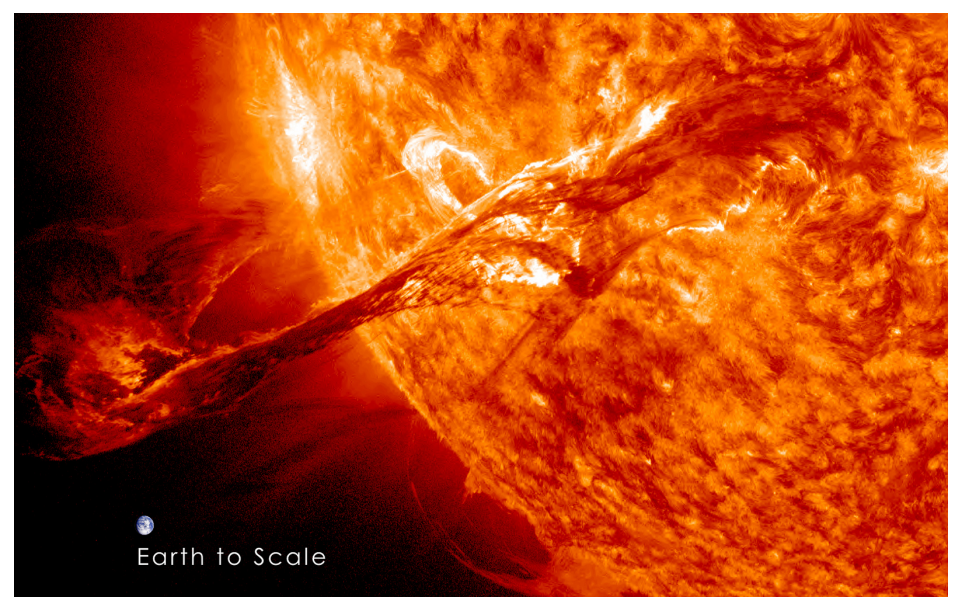




\section{What drives mass loss?}

Nasa.gov

\section{Stellar Winds}

Primary Mechanism

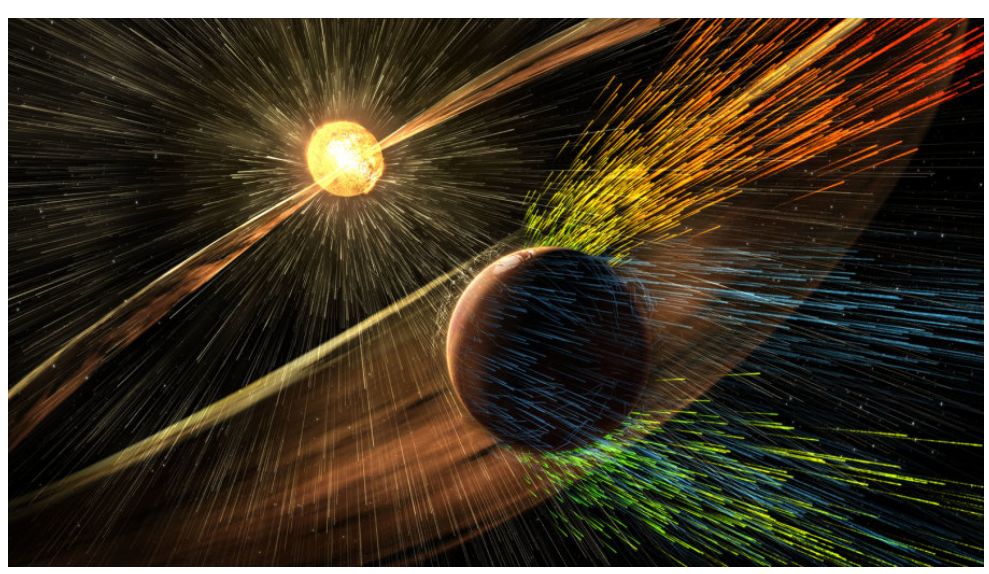

Coronal Mass Ejections (CMEs)

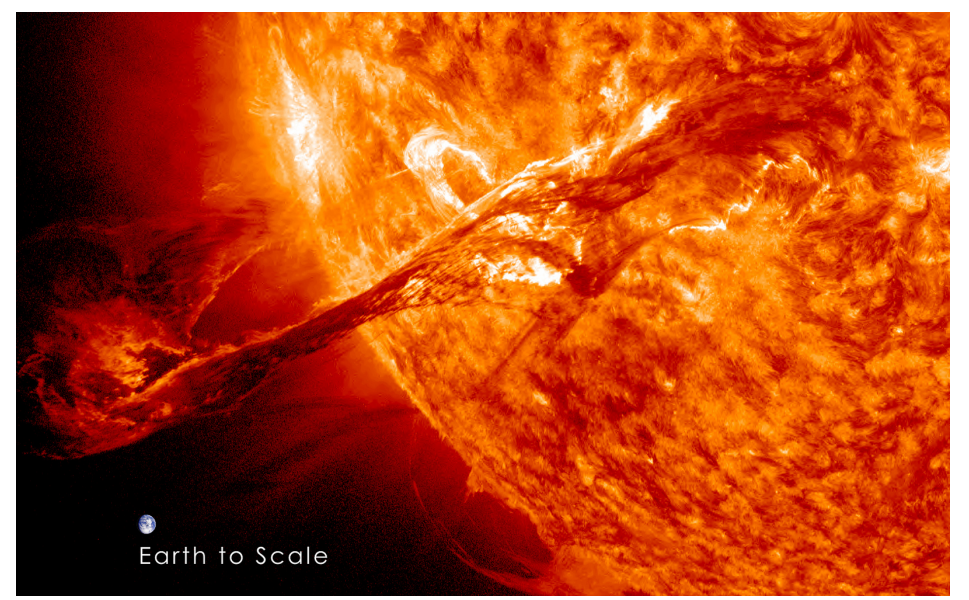




\section{What powers stellar winds?}

Solar-type stars have a corona, made of hot plasma $\sim 2$ Million ${ }^{\circ} \mathrm{K}$

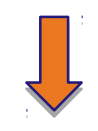

Magnetic activity on the stellar surface drives waves called Alfvén waves

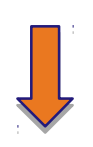

Alfvén waves heat the stellar corona

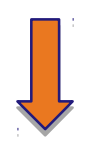

A hot corona produces X-rays and stellar wind 
Los Alamos National Laboratory

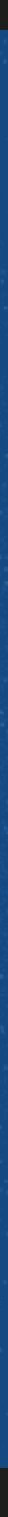




\section{Stellar wind strength is tied to coronal X-ray activity}

Observations of other solar-type stars tell us how stellar winds scale with coronal activity

$$
\dot{M} \propto L_{X}
$$

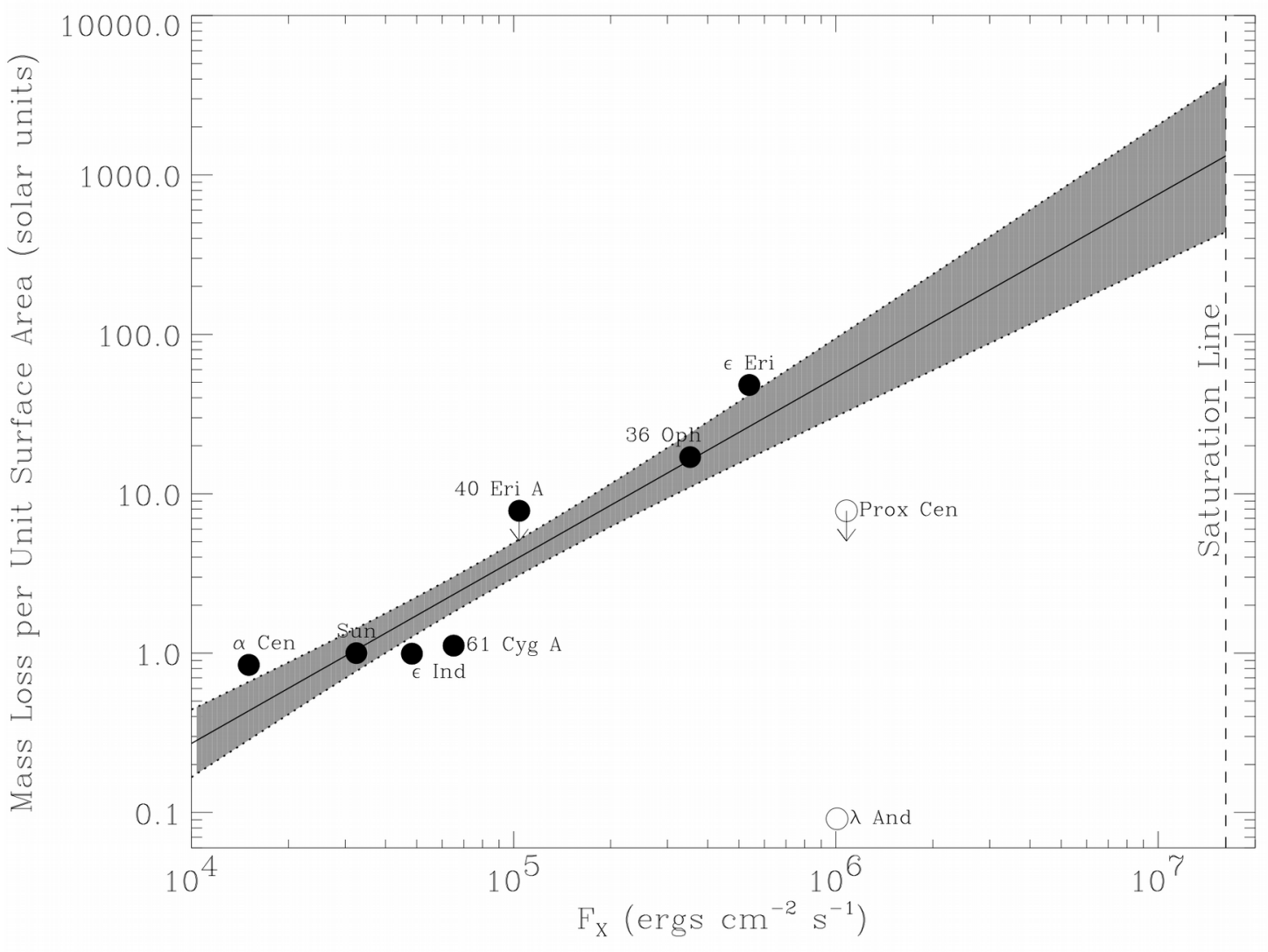

(Wood et al. 2005) 


\section{Mass loss estimates by R. Wood}
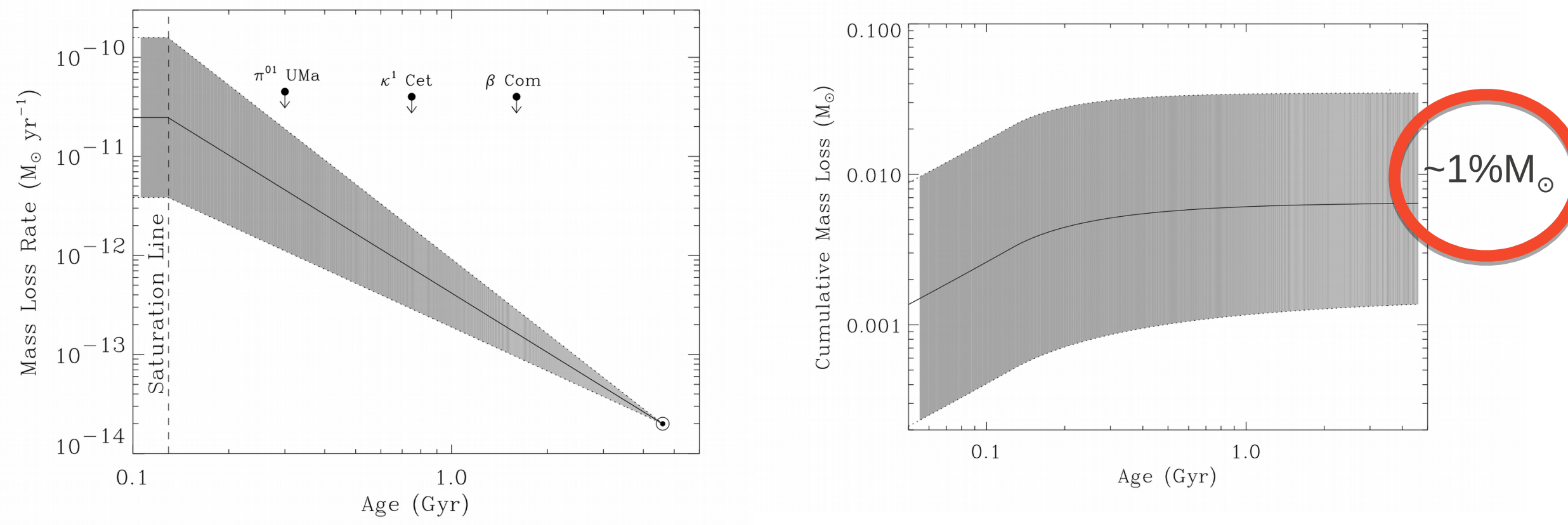

(Wood et al. 2005) 


\section{What if the Sun was much more magnetically active early in its life?}




\section{What if the Sun was much more magnetically active?}

- Stronger magnetic fields

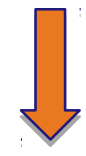

- More coronal heating (from Alfvén waves)

- More X-rays

- More mass loss 
What does the Sun's magnetic field strength depend on?

\author{
Convective motions
}

$$
\text { \& }
$$

The solar rotation rate 
Fast movement of plasma in the convection zone produces magnetic fields

We characterize the speed of these motions with a parameter called the convective turnover time
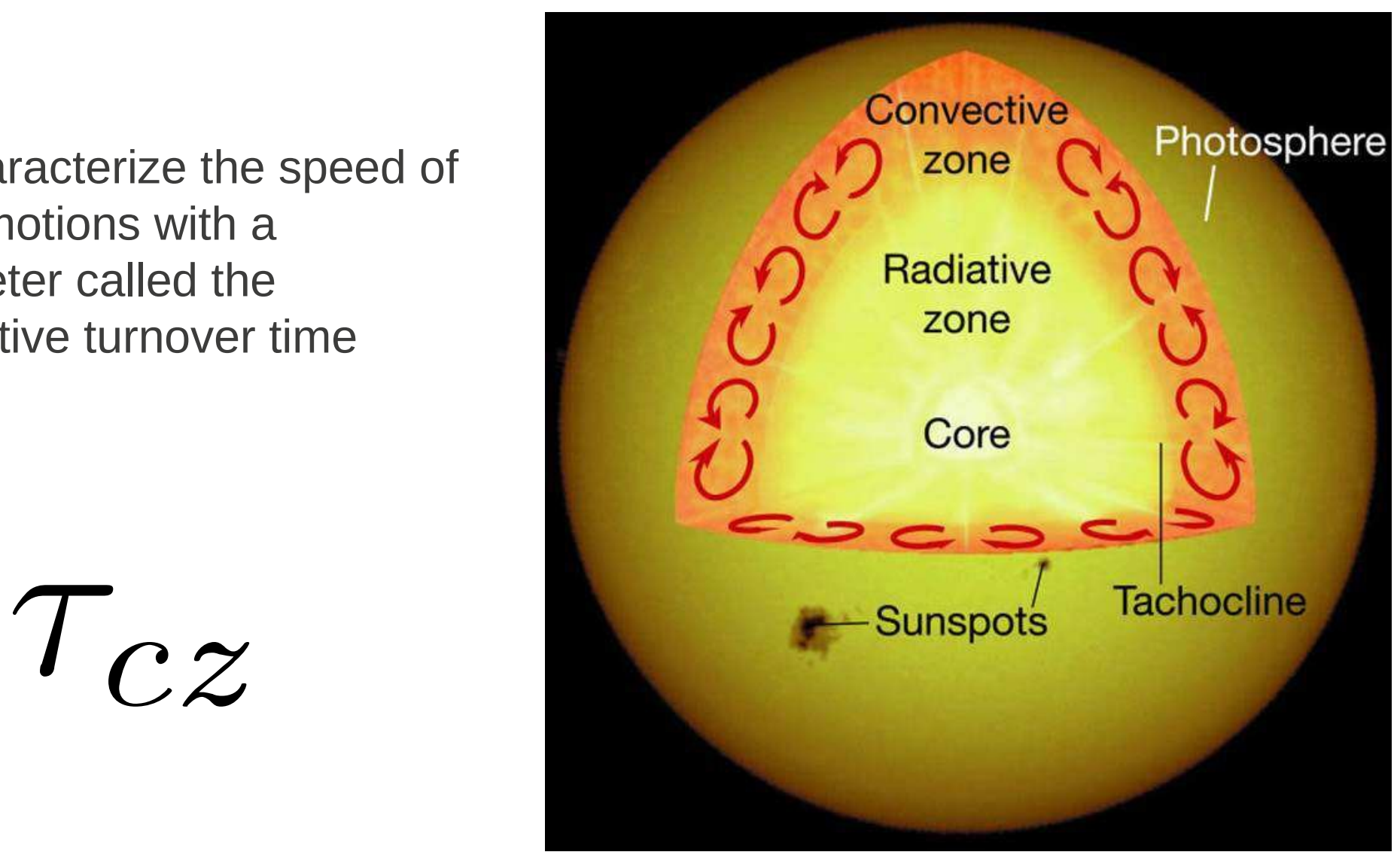
Rotation of the Sun also produces magnetic fields

We characterize the speed of the Sun's rotation with a parameter called the rotation Period
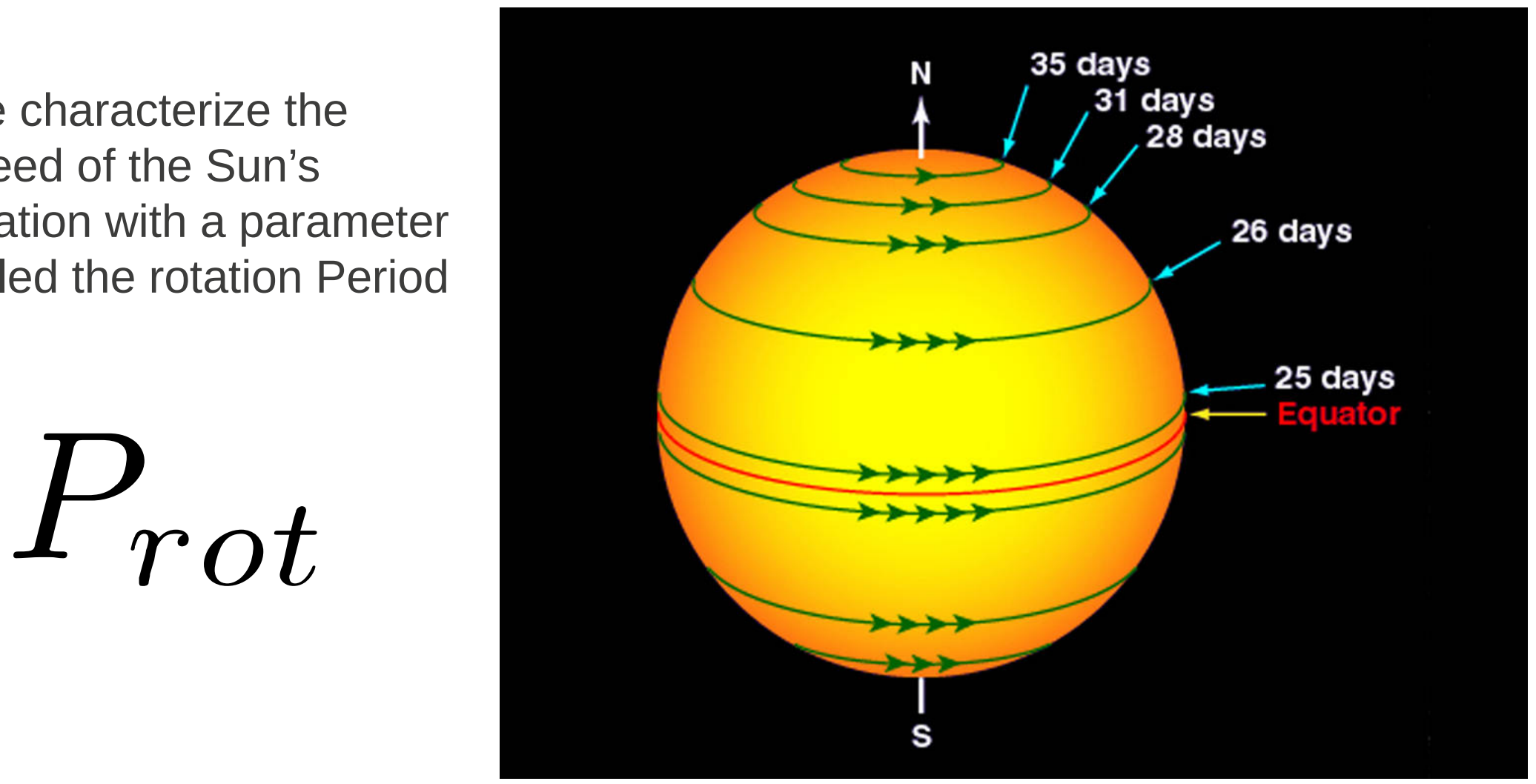

Nasa.gov 


\section{We combine both parameters into one called the Rossby number}

$$
R_{o}=\frac{P_{r o t}}{\tau_{c z}}
$$

The Rossby number is dimensionless number that represents the ratio of inertial forces to Coriolis forces 


\section{Stellar activity is a function of the Rossby number}

$$
R_{X}=L_{X} / L_{b o l}
$$

$$
R o=\frac{P_{r o t}}{\tau_{c z}}
$$

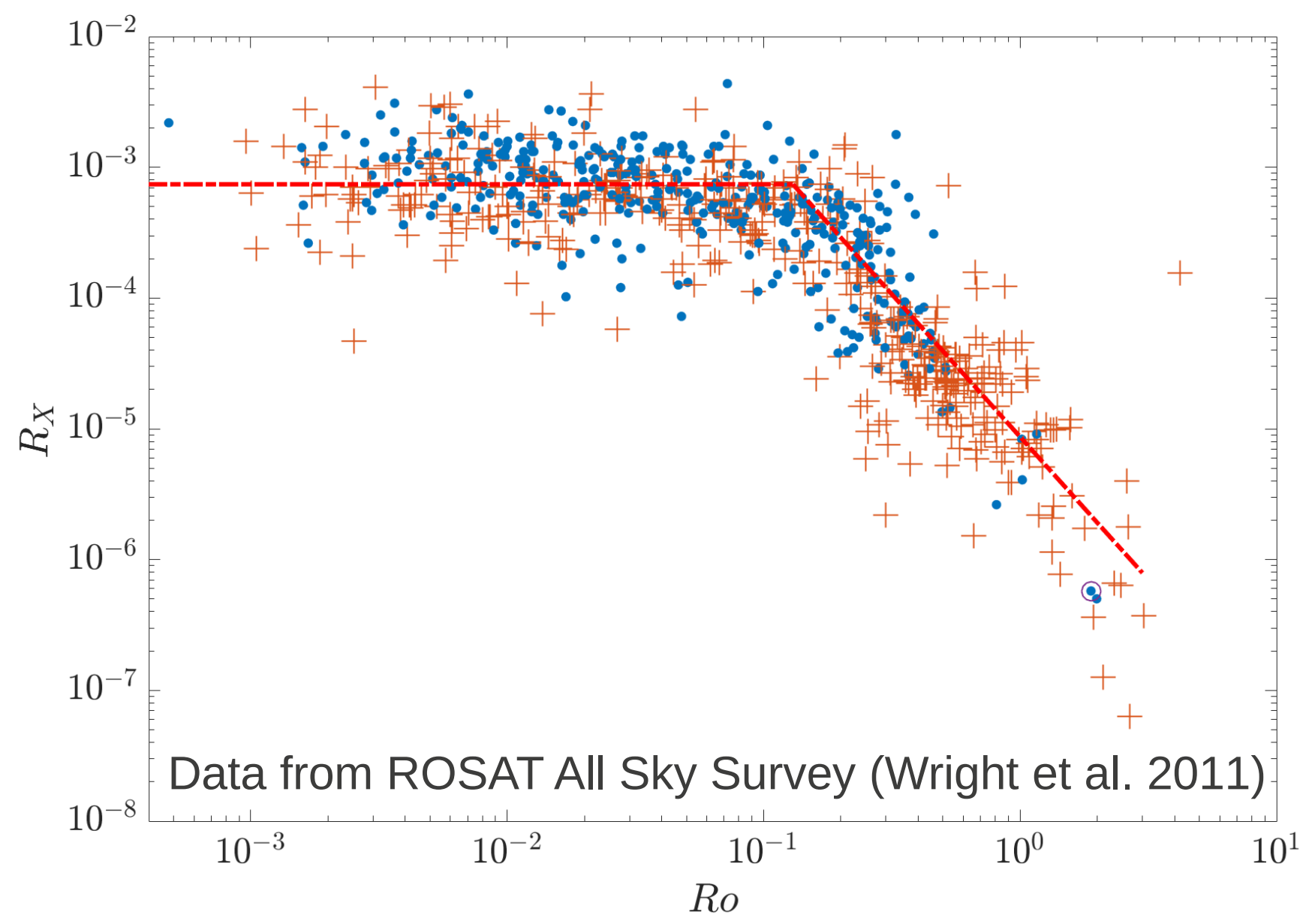


When the Rossby number becomes too low, the star's activity saturates

$$
\begin{gathered}
R_{X}=L_{X} / L_{b o l} \\
R o=\frac{P_{r o t}}{\tau_{c z}}
\end{gathered}
$$

\section{But why?}

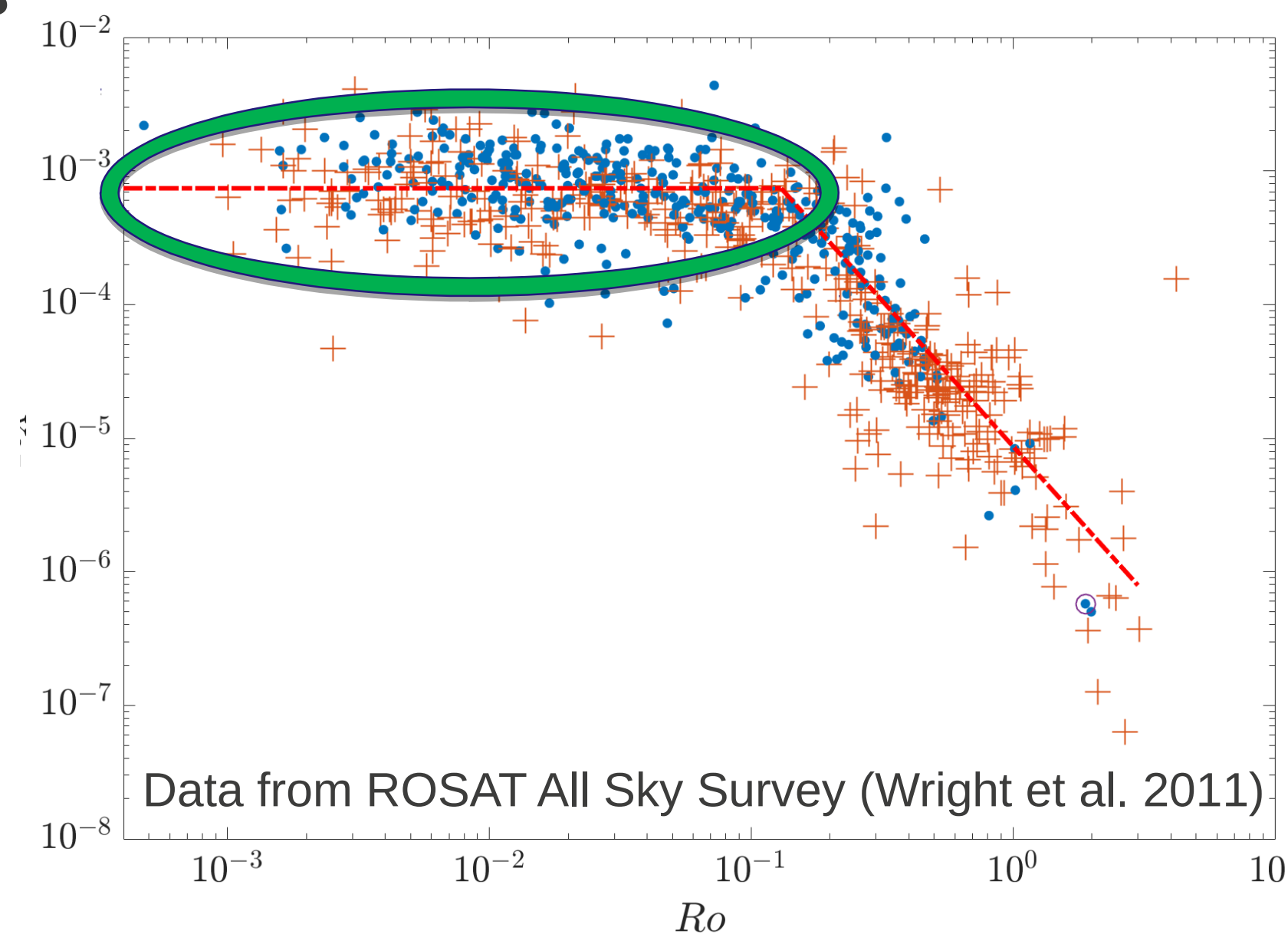


Why does a solar-type star's activity saturate at low Rossby numbers?

\section{Star spots}

$\&$

Magnetic feedback from shearing 


\title{
A low Rossby number leads to more star spots
}

\author{
If a star is more magnetically active
}

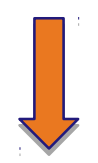

It will have more star spots

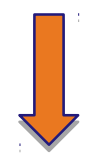

If a star's surface becomes covered in star spots, the total magnetic field strength will begin to saturate 


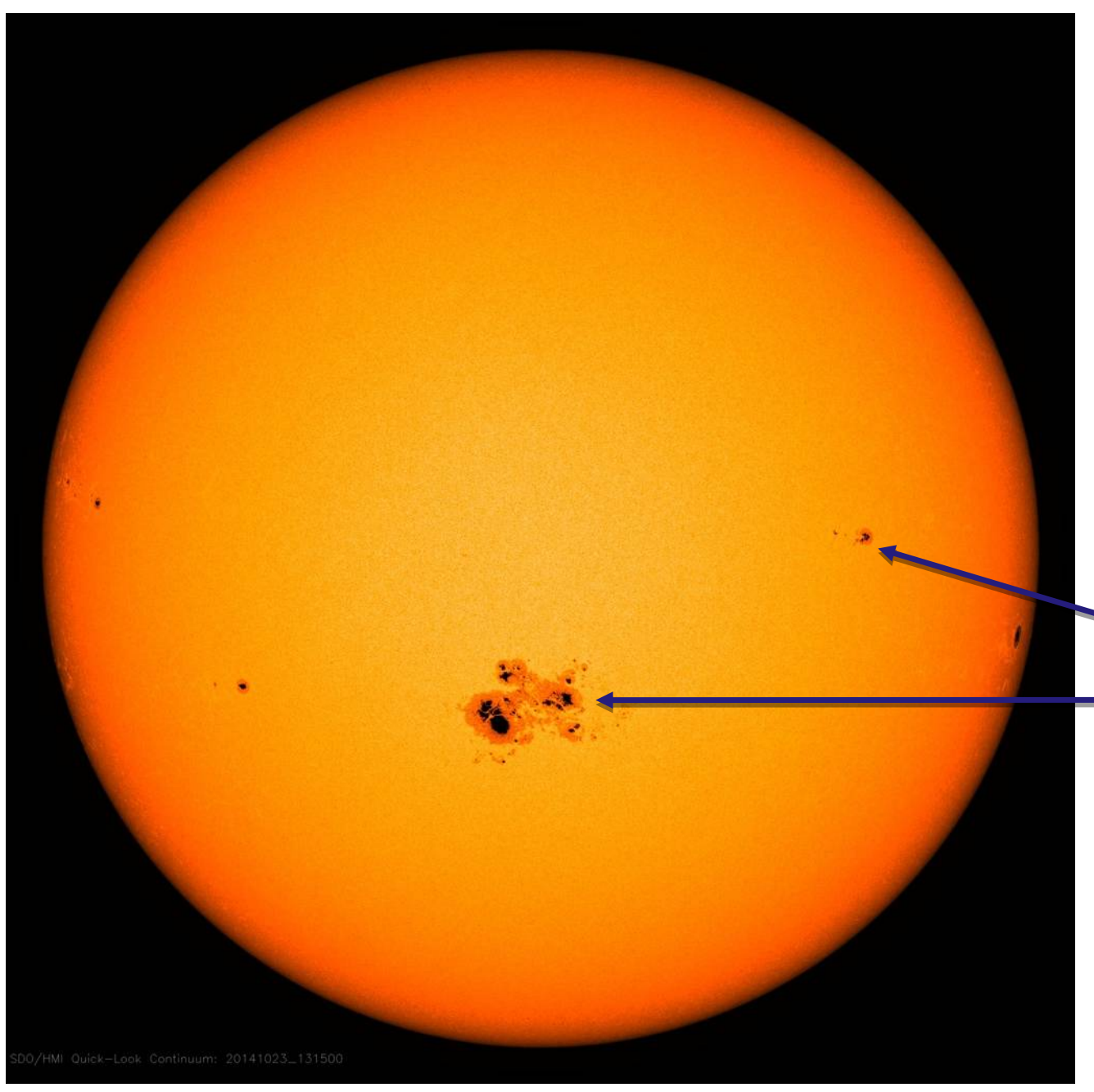

Sun spots

(Namekata et al. 2018) 


\section{A low Rossby number leads to strong magnetic feedback}

We assume a solar-like dynamo where the shear (pole to equator) correlates with the rotation rate

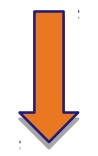

At high rotation rate, the rotation can have a strong Coriolis force that can suppress differential rotation

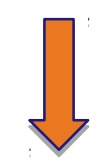

Suppressing of differential rotation limits magnetic field strength 


\section{Recap}

- Faster rotation (low Ro) $\longrightarrow R o=\frac{P_{r o t}}{\tau_{c z}}$

- Larger shear and winding of the field

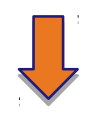

- Stronger magnetic field

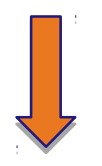

- More coronal heating (from Alfvén waves)

- More X-rays (up to a saturation threshold)

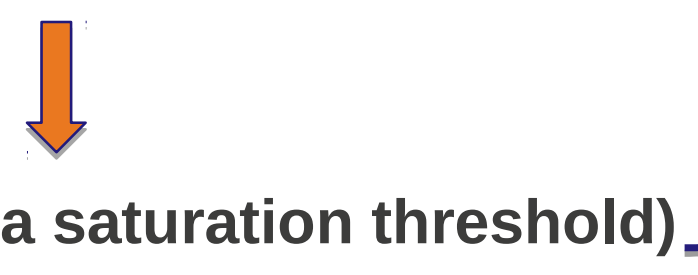




\section{Stellar Winds extract angular momentum from stars}

Mass loss via stellar wind

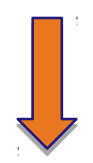

Stellar wind carries away angular momentum

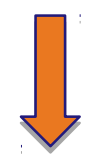

Star spins down

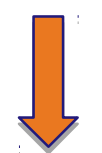

Accurate models of solar winds must reproduce the current solar mass loss rate and rotation rate 


\title{
How do we model this?
}

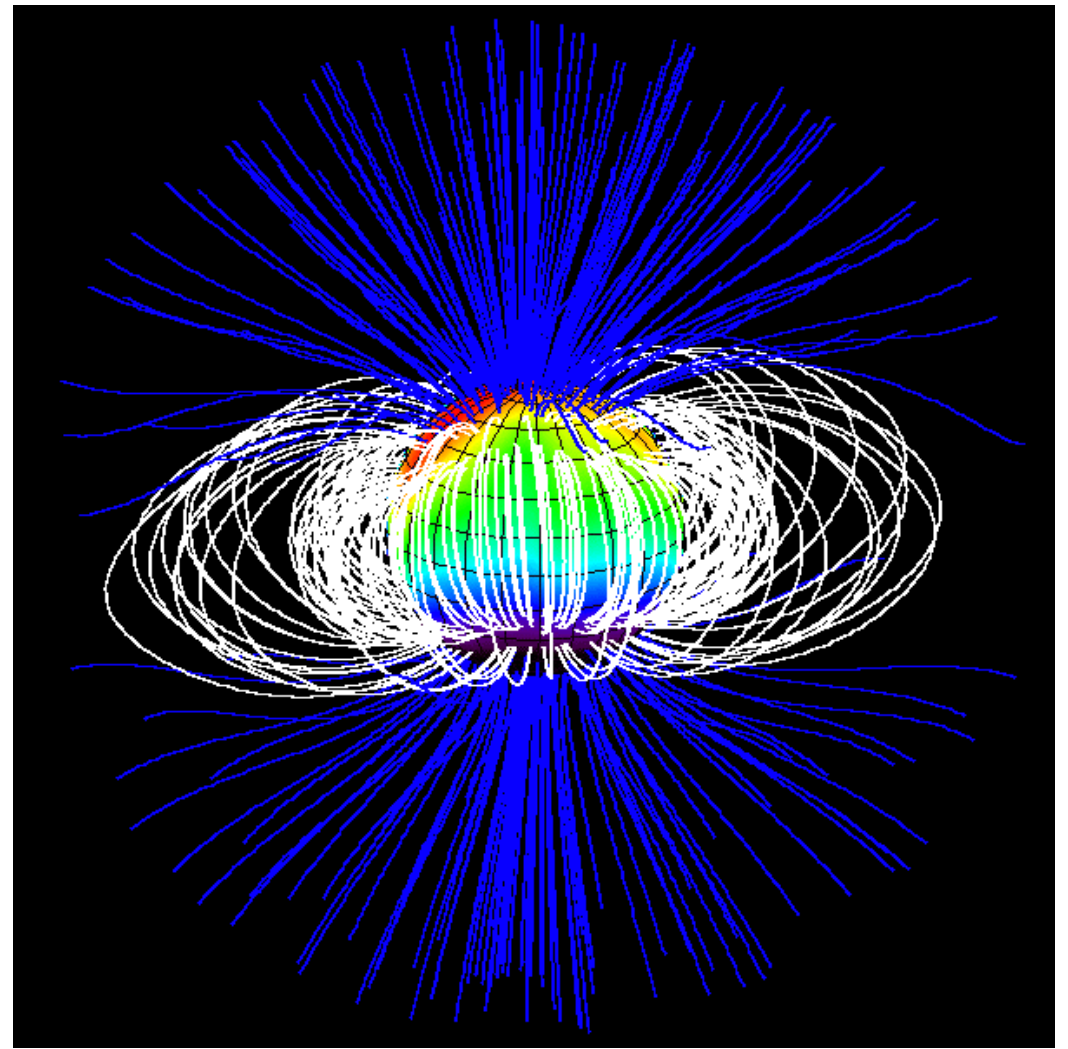

MM Jardine \& J-F Donati

\author{
MM Jardine \& J-F Donati
}




\section{We trace the feedback between rotation and magnetized stellar winds}

Magnetically driver solar winds extract angular momentum from the star (magnetic braking) This decreases the rotation rate of the star, which in turn decreases the field strength. 


\section{Remember this figure?}

$$
\begin{gathered}
R_{X}=L_{X} / L_{b o l} \\
\downarrow \\
\dot{M} \propto L_{X}
\end{gathered}
$$

$$
R o=\frac{P_{r o t}}{\tau_{c z}}
$$

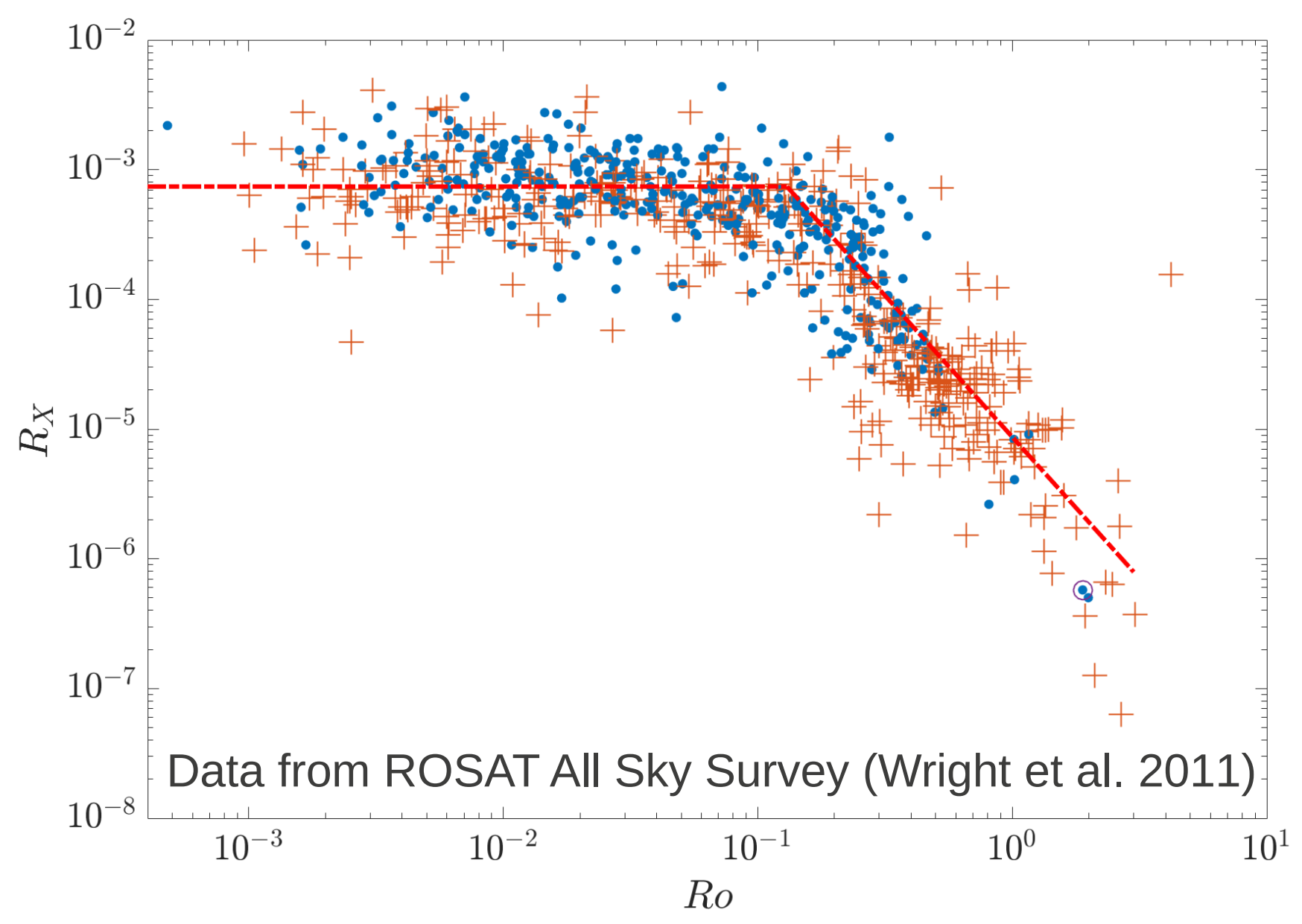




\section{We explore variations in the activity-rotation relationship}

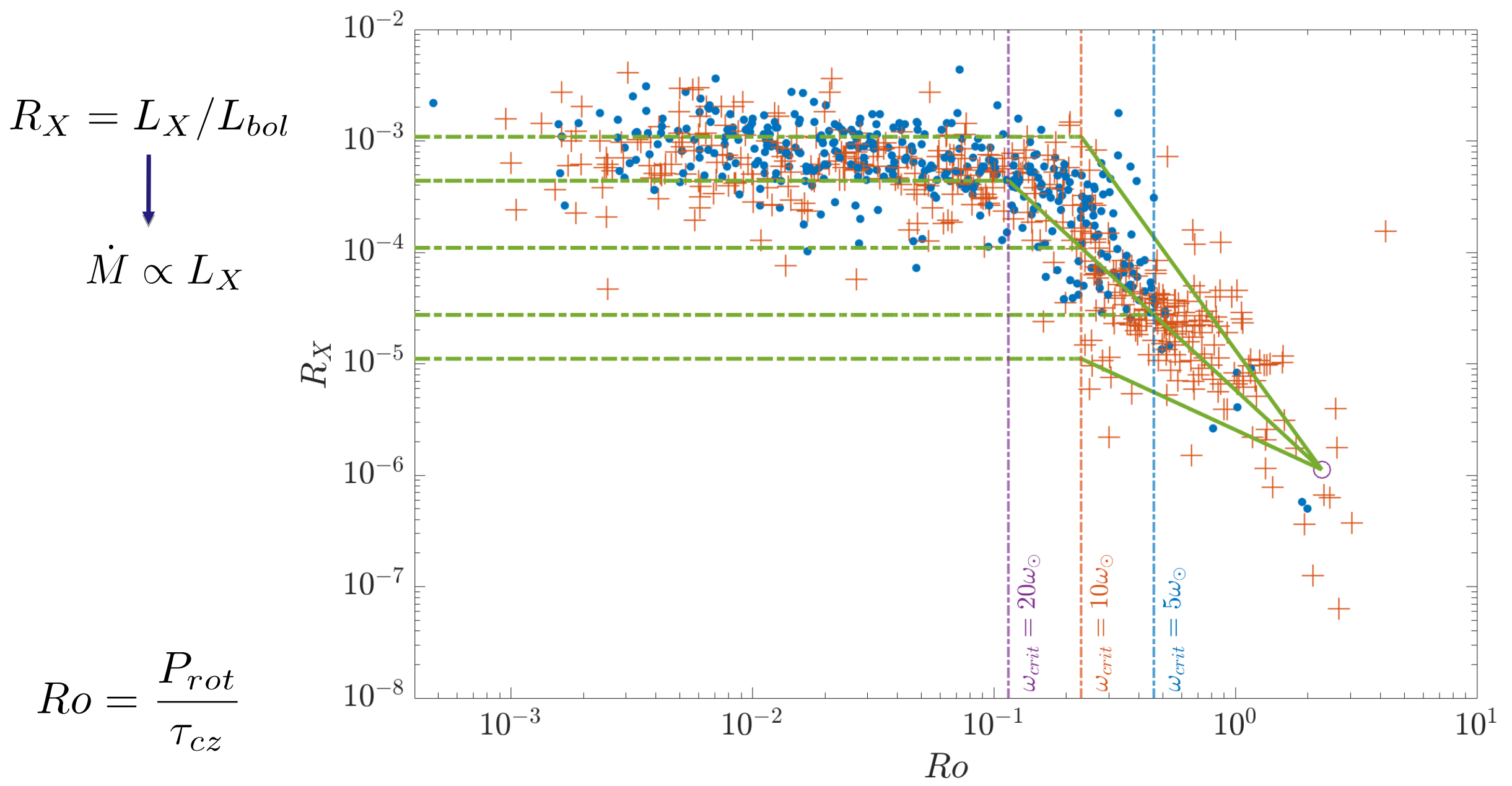




\section{We explore variations in the activity-rotation relationship}

- 3 different spin-rate thresholds $\left(\omega_{\text {crit }}\right)$ for magnetic field saturation (fully covered in spots)

- Critical spin rate of:

$$
\omega_{\text {crit }}=5 \omega_{\odot}, 10 \omega_{\odot}, 20 \omega_{\odot} ; \quad a=2
$$

- 3 different scaling relations between magnetic field strength and mass loss(a)

- Linear, square, and cubic relationships

$$
a=1,2,3 ; \quad \omega_{\text {crit }}=10 \omega_{\odot}
$$




\section{We Compute The Torque On The Star From Magnetized Stellar Winds}

$$
\begin{aligned}
& \tau_{w}=\frac{K_{1}^{2}}{(2 G)^{m}} B_{*}^{4 m} \dot{M}_{w}^{1-2 m} \frac{R_{*}^{5 m+2}}{M_{*}^{m}} \frac{\omega_{*}}{\left(K^{2}+0.5 f^{2}\right)^{m}} \\
& f \equiv \omega_{*} R_{*}^{3 / 2}\left(G M_{*}\right)^{-1 / 2} \\
& m=0.22 \\
& K_{2}=0.0506 \\
& K_{1} \equiv f_{K}=\text { Fitting Parameter }
\end{aligned}
$$

(Matt et al. 2012) 


\section{Angular Momentum Formulation}

$$
\begin{aligned}
R_{X} & =L_{X} / L_{b o l} \sim \omega^{a} & B_{*} \sim\left(\omega_{*}\right)^{b} P_{p h o t}^{1 / 2} \\
\dot{M} & \propto L_{X} \quad \text { (Wood et al. 2005) } & b=1
\end{aligned}
$$

$$
\frac{d J}{d t}= \begin{cases}f_{K} K_{M} \omega\left(\frac{\omega_{c r i t}}{\omega_{\odot}}\right)^{a(1-2 m)+4 m b} & \omega_{c r i t} \leq \omega \frac{\tau_{c z}}{\tau_{c z, \odot}} \\ f_{K} K_{M} \omega\left(\frac{\omega \tau_{c z}}{\omega_{\odot} \tau_{c z, \odot}}\right)^{a(1-2 m)+4 m b} & \omega_{c r i t}>\omega \frac{\tau_{c z}}{\tau_{c z, \odot}}\end{cases}
$$

$$
\frac{K_{M}}{K_{M, \odot}}=c(\omega)\left(\frac{R}{R_{\odot}}\right)^{5 m+2}\left(\frac{M}{M_{\odot}}\right)^{-m}\left(\frac{L}{L_{\odot}}\right)^{1-2 m}\left(\frac{P_{p h o t}}{P_{p h o t, \odot}}\right)^{2 m}
$$

(Farag \& Pinsonneault, in prep, 2019) 


\section{Variations in initial conditions}

- Stars form with various initial rotation periods

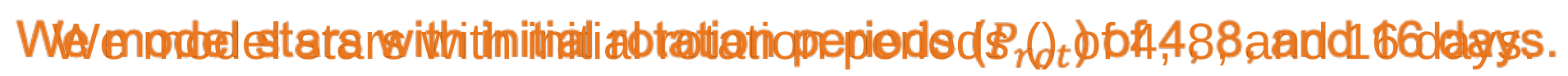

- Proto-planetary discs interact with forming stars, effectively maintaining the star's initial rotation period

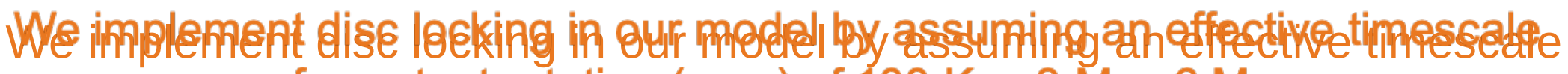

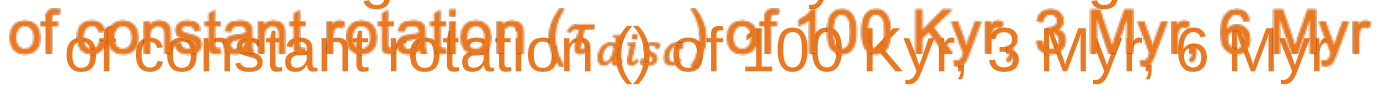




\section{The following slides are results of our Calculations}

We compare our results to solar type stars from various clusters of various known ages.

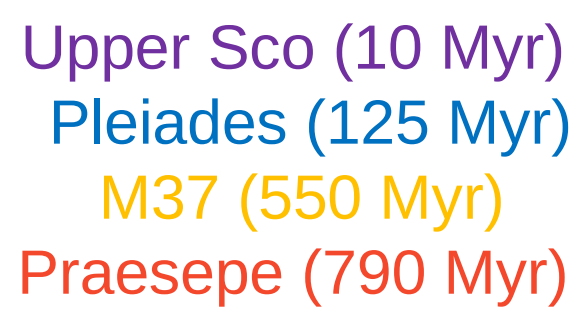

We specifically look at stars within $5 \%$ the mass of the Sun 


\section{Predicted Rotation Histories}

Weak scaling

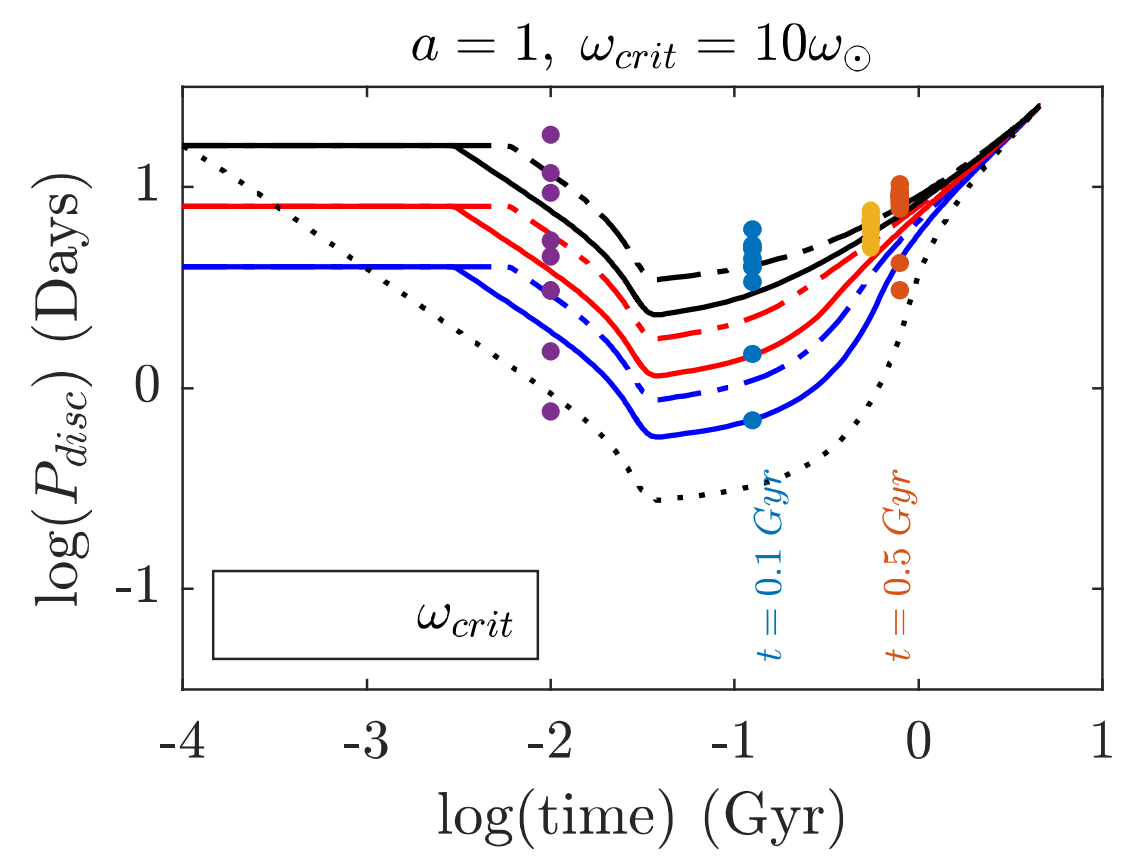

These Models Slightly Over-Predict The Rotation Periods

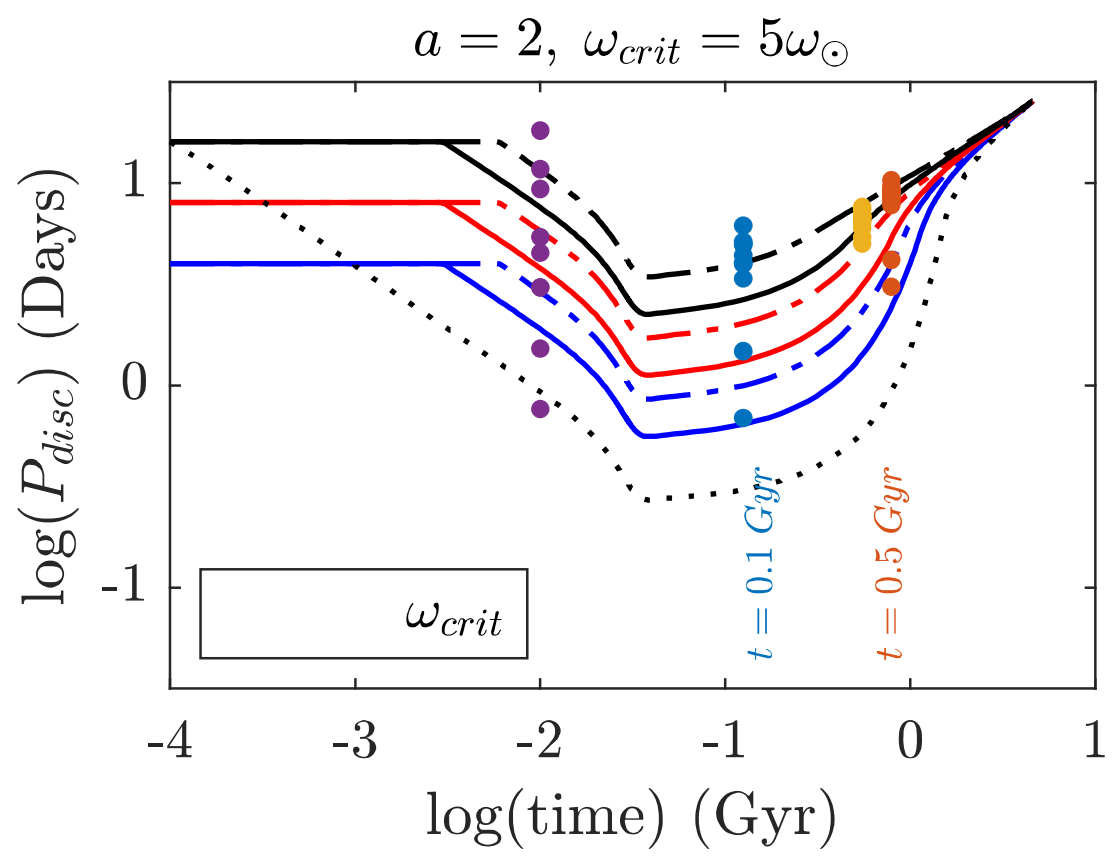

Upper Sco (10 Myr) Pleiades (125 Myr) M37 (550 Myr) Praesepe (790 Myr) 


\section{Predicted Rotation Histories} Strong scaling

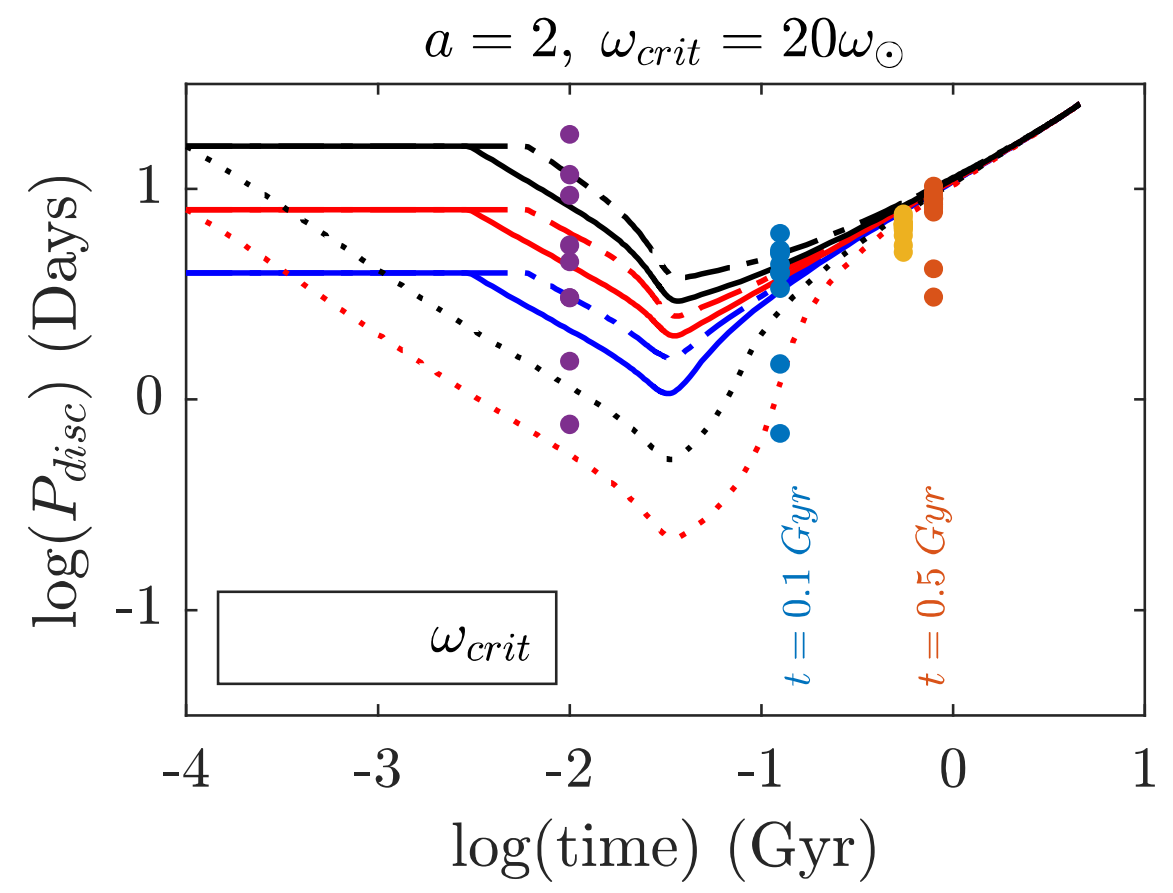

These Models Under-Predict The Rotation Periods

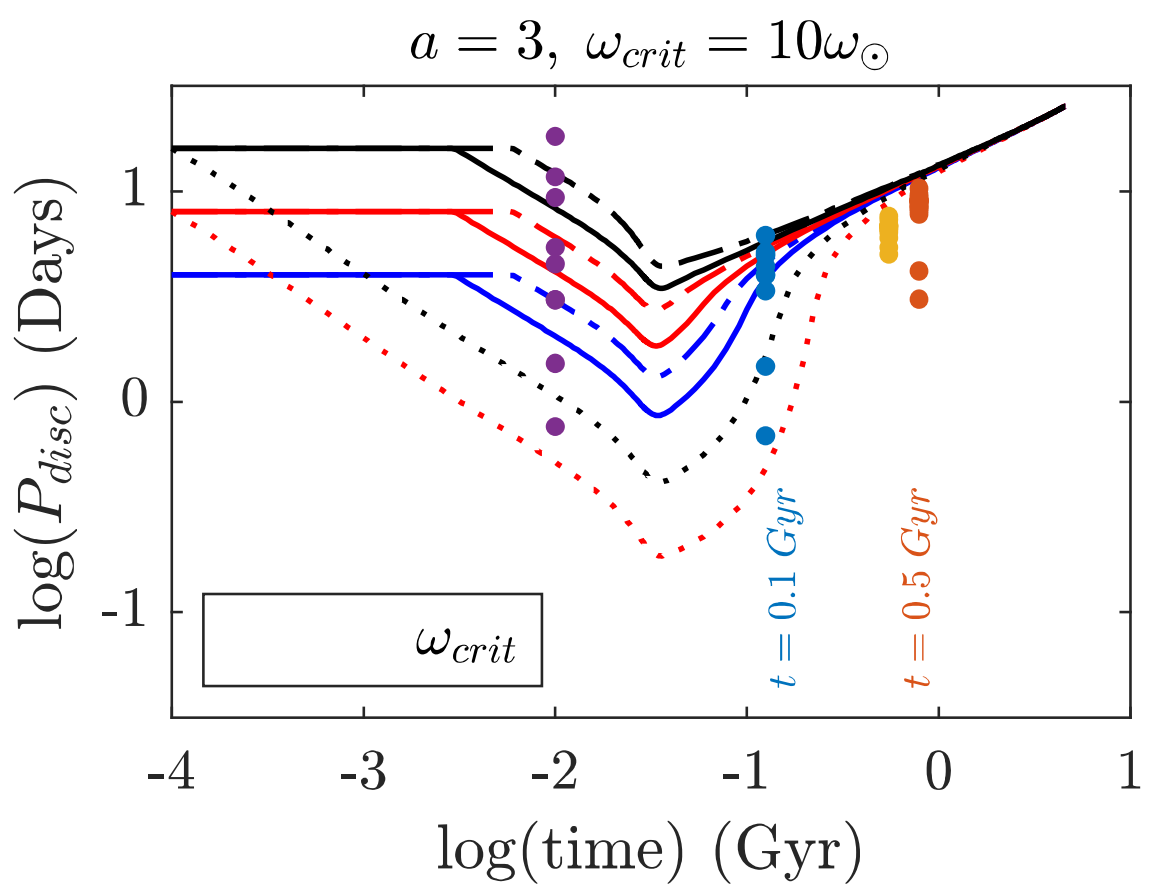

Upper Sco (10 Myr) Pleiades (125 Myr) M37 (550 Myr) Praesepe (790 Myr) 


\section{Predicted Rotation Histories Moderate scaling}

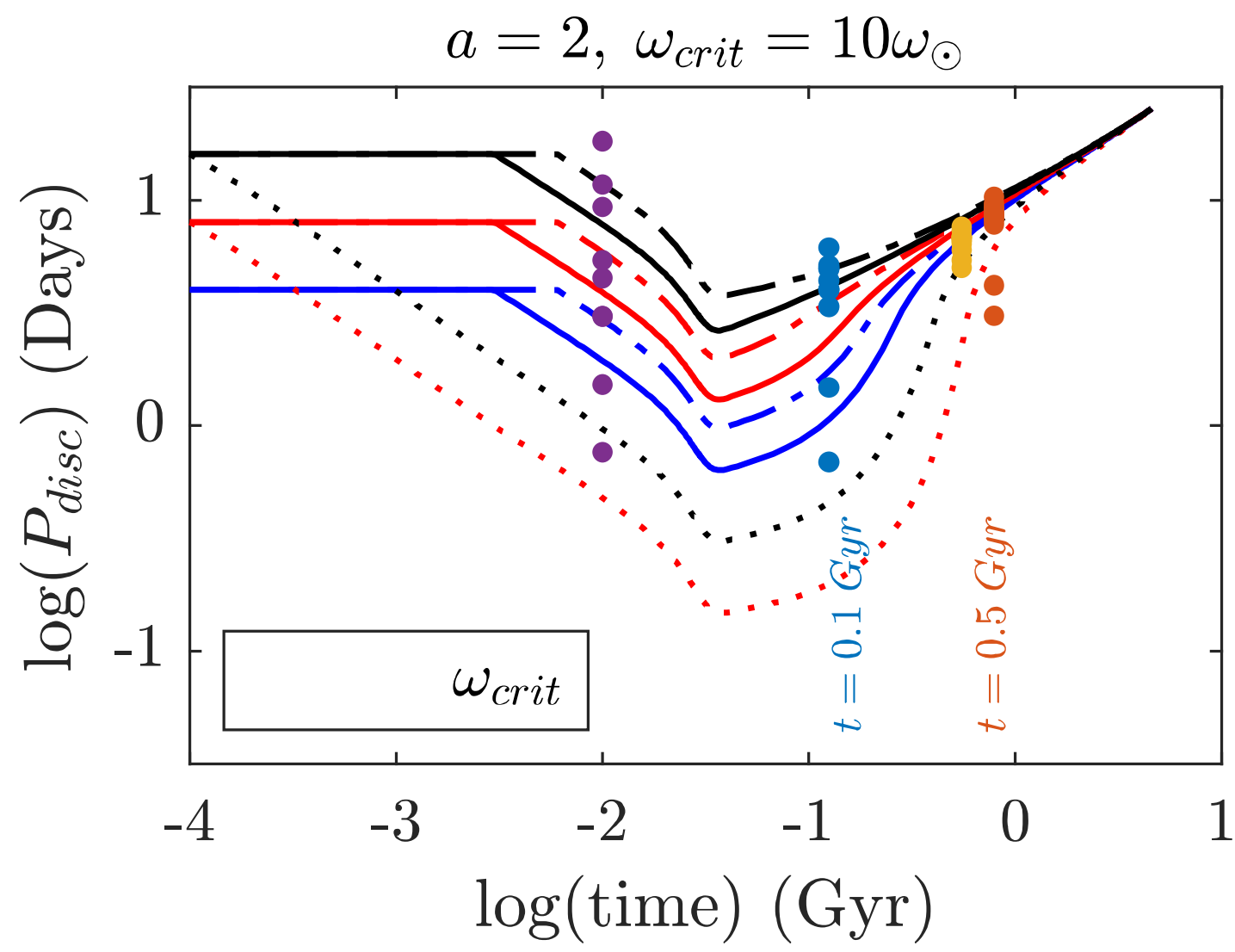

Reasonably Good Agreement

Upper Sco (10 Myr) Pleiades (125 Myr) M37 (550 Myr)

Praesepe (790 Myr) 


\section{Key results of our predicted rotation histories}

1. All solar-type stars spin down very early in their evolution.

2. If a star is very active, it will not remain active for much of its lifetime. 
If You Adopt Our Rotation Histories, You Can Predict Mass Loss Rates 
Weak scaling
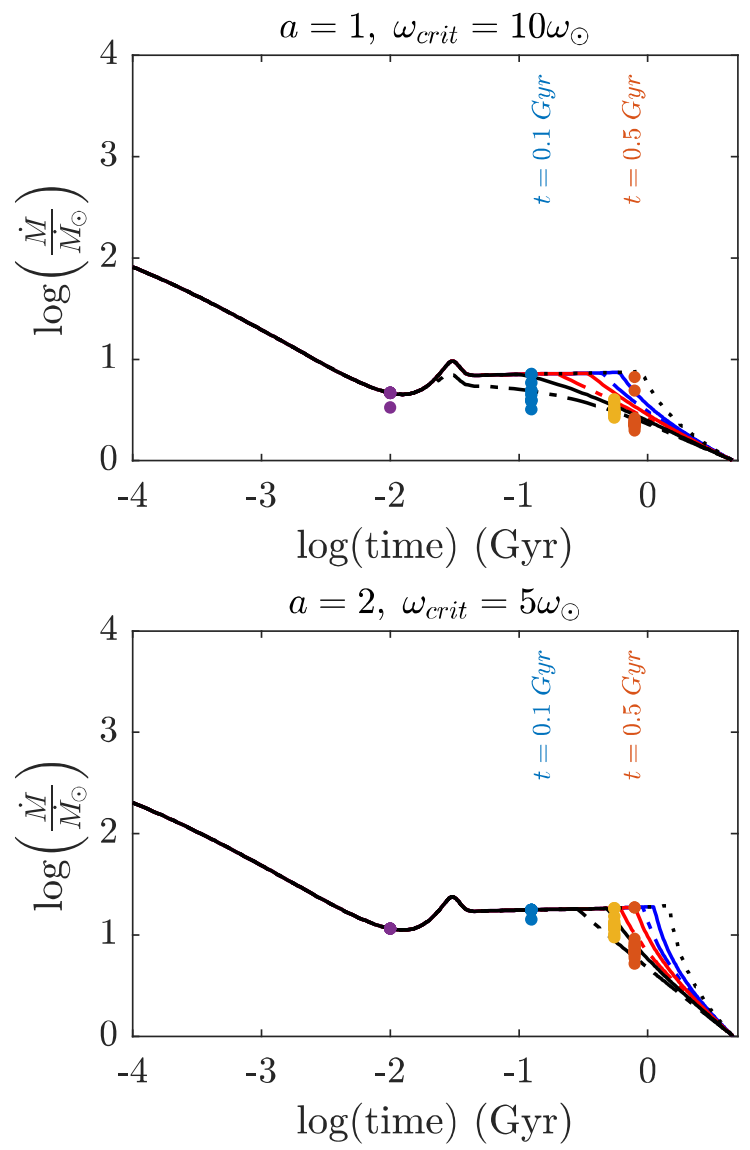

\section{Mass Loss Rate Histories}

Moderate scaling

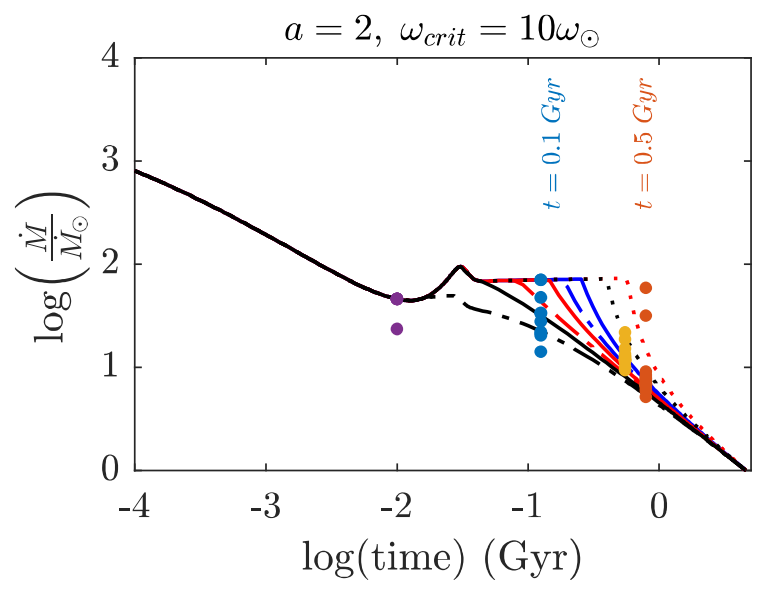

Upper Sco (10 Myr) Pleiades (125 Myr) M37 (550 Myr)

Praesepe (790 Myr)
Strong scaling
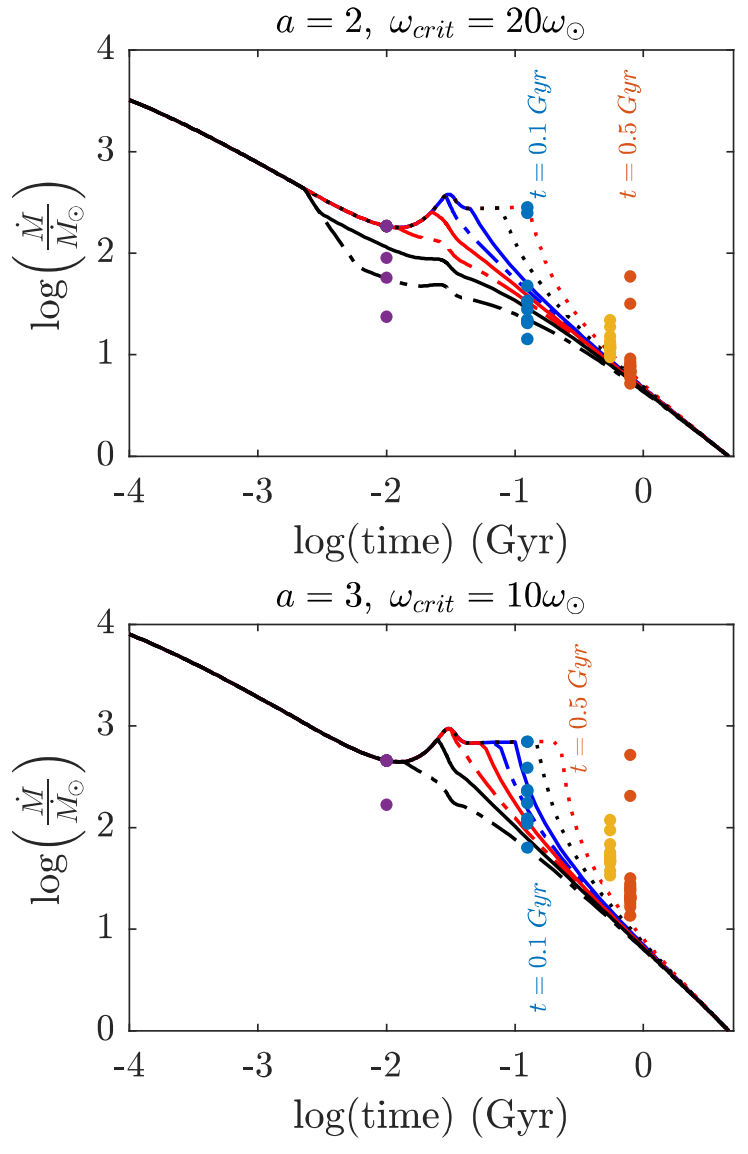


\section{Key results of our predicted mass loss histories}

All models predict very short-lived timescales of high mass loss, with most occurring within the first $\sim 100 \mathrm{Myr}$.

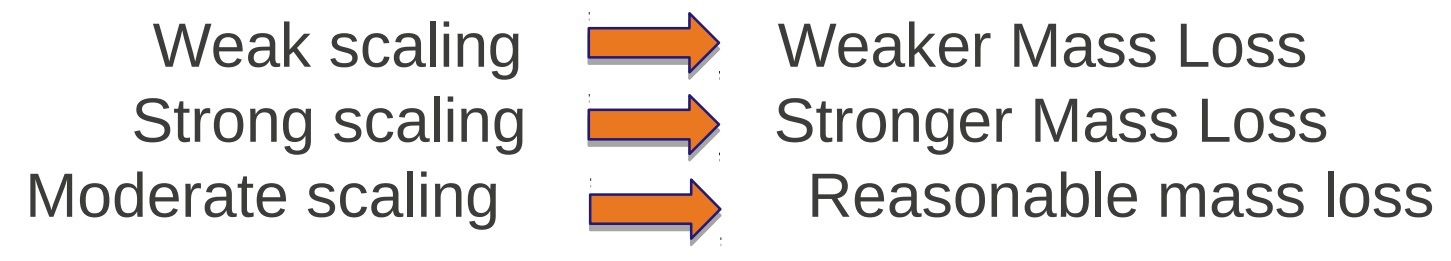

We have strong feedback in the system that strictly limits the early mass loss history of the Sun. 


\section{Nominal case}

Moderate scaling
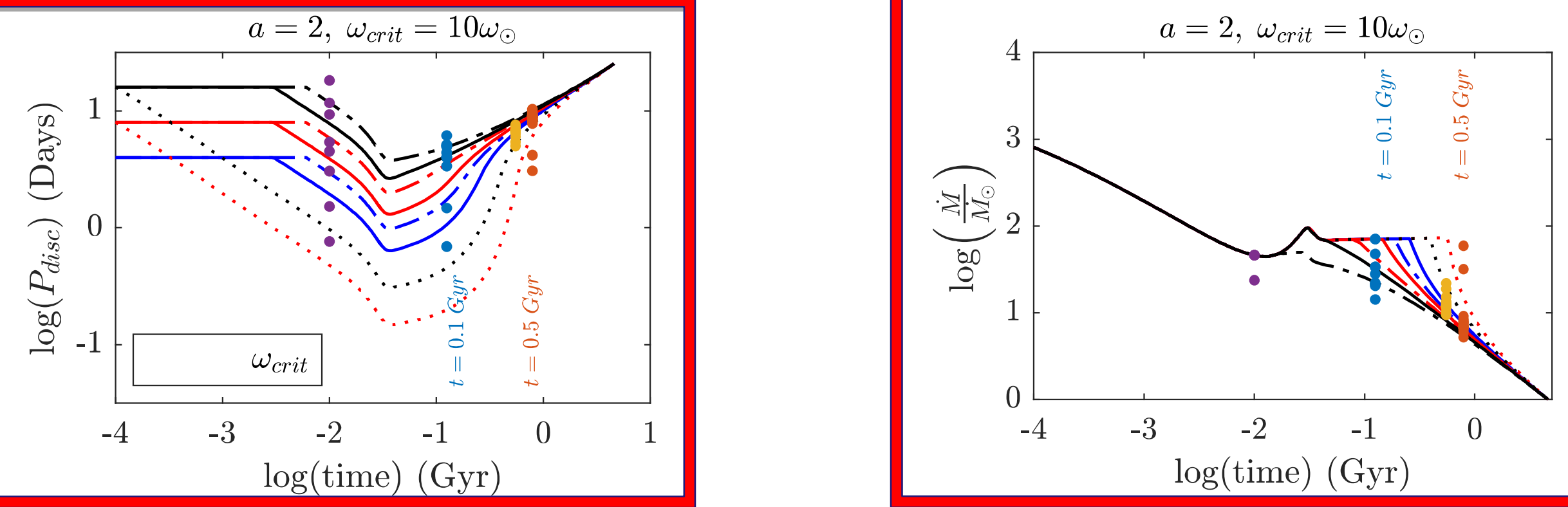

Upper Sco (10 Myr)

Pleiades (125 Myr)

M37 (550 Myr)

Praesepe (790 Myr) 


\section{Total mass loss}

We integrate the mass loss histories for each of our physical cases to predict total mass loss 


\section{Total mass loss}

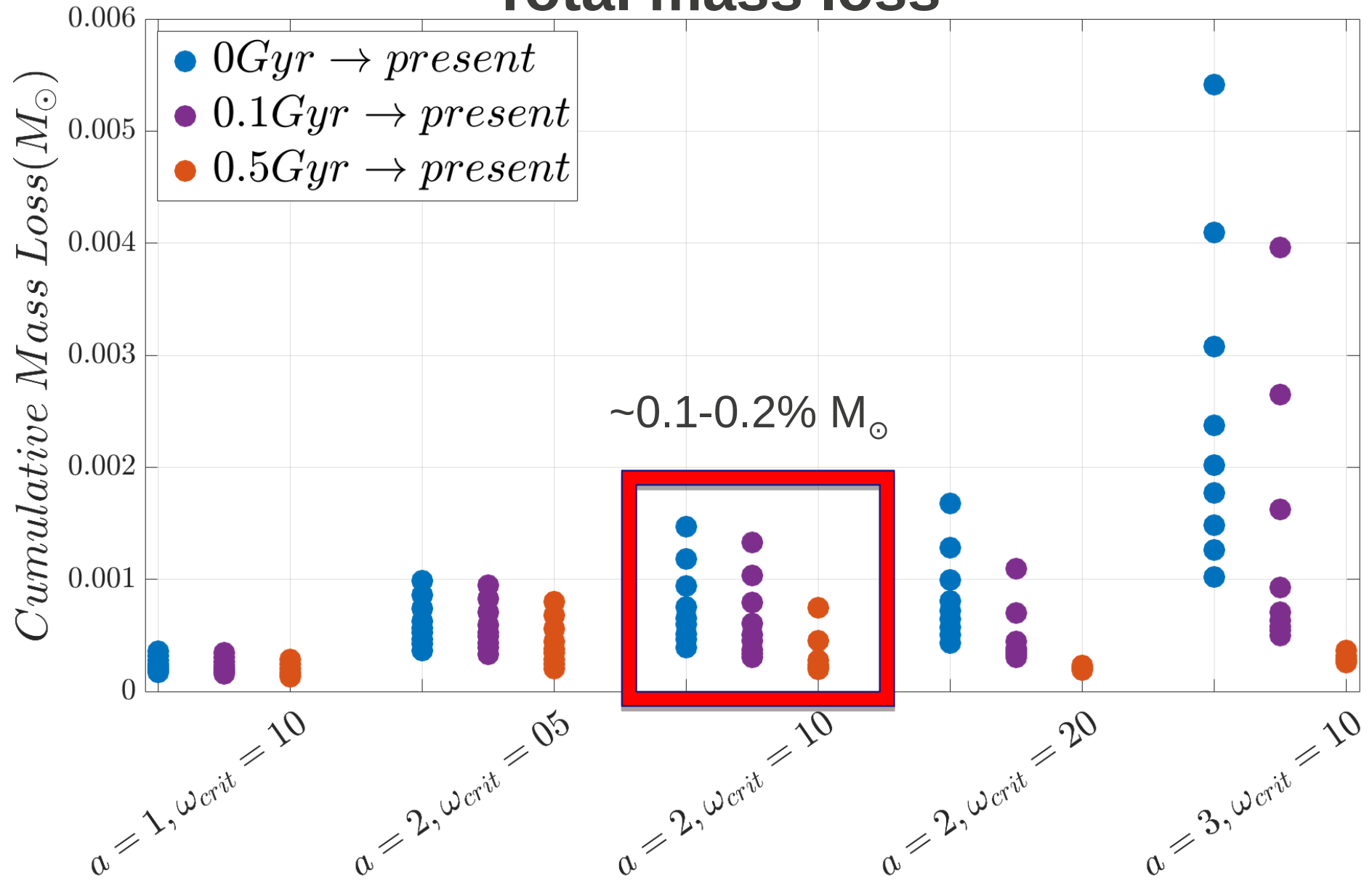


Let's look back at our luminosity and mass plots!
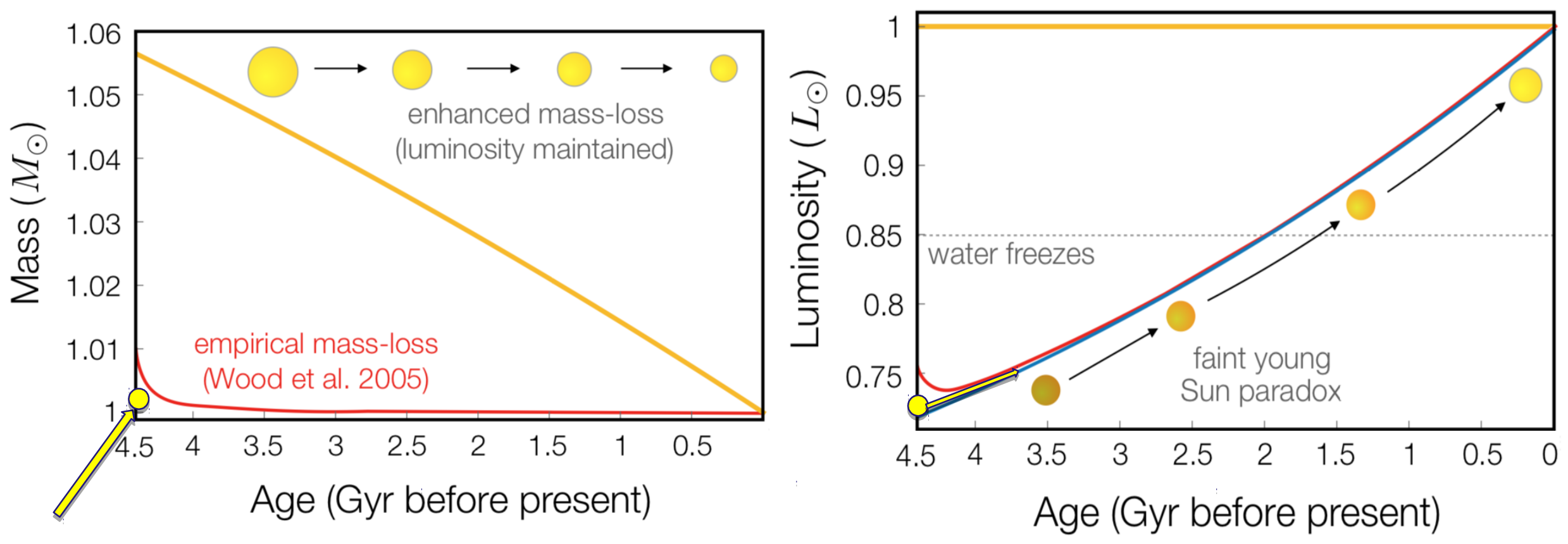

The Sun $y$ is more ma
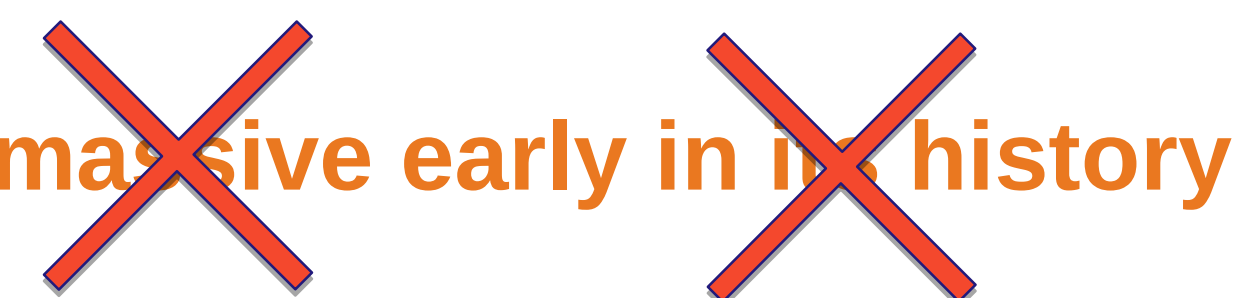


\section{Other possible solutions to the FYSP}

- Earth

- A faster spinning earth could enhances the equator-to-pole temperature gradient, facilitating a warm equator, while maintaining cold poles. "The greenhouse warming required to generate an ice-free fast spinning Earth can differ from that required to generate an Earth with permanent ice caps by the equivalent of 1-2 orders of magnitude of pCO2." (Spalding et al. 2019)

- Mars

- Advanced simulations show that water on Mars could have leaked out of glacier on geologically short timescales of 100-1000 years and frozen over again (Palumbo et al. 2019). 


\section{Summary and Conclusion}

- Faint Young Sun Paradox : a more massive Sun has been proposed as a solution

- We have calculated possible solar rotation histories and determined reasonable estimates for the total mass shed off by the star over its lifetime

- We predict that all solar-type stars spin down very early in their evolution, are therefore inactive, and as a result have low mass loss

- The Sun was probably $0.1-0.2 \%$ more massive than it is today

- Better solutions to the Faint Young Sun Paradox, from more advanced models of early Earth and Mars, are needed 


\section{Questions?}

